\title{
EXTREMELY HIGH FREQUENCY RESOLUTION AND LOW HARMONIC DISTORTION DIGITAL LOOK-UP-TABLE SINUSOIDAL OSCILLATORS
}

\author{
M. M. AL-IBRAHIM (JARRAH)* \\ Electrical Engineering Department, King Fahd University of Petroleum \\ and Minerals, Dhahran 31261, P.O. Box 822, Saudi Arabia
}

(Received 21 June 2000; In final form 12 July 2000)

\begin{abstract}
New techniques to efficiently increase the frequency resolution of digital sinusoidal oscillators based on look-up-table (LUT) methods are proposed. The increase in frequency resolution is achieved while maintaining very low level of spurious harmonic distortion. The proposed techniques increase the LUT length to a level at which the spurious harmonic distortion is negligible. The first proposed technique is based on partitioning the address register into three sets and dividing the available LUT length into three smaller tables addressed according to the content of the address register sets. The second proposed technique utilizes one LUT and interpolates the values of the samples that are not stored in the table. The third proposed technique is similar to the first technique with the advantage of simpler implementation and lower levels of spurious harmonic distortion. The proposed techniques are simulated and their performance is compared with that of the direct LUT and trigonometric interpolation methods. The simulation results show that the proposed techniques are superior to both direct LUT and trigonometric interpolation methods.
\end{abstract}

Keywords: Harmonic distortion; Frequency resolution; Digital oscillators; Look-uptable; Fractional addressing; Interpolation methods

*Present address: Jordan University of Science and Technology, Irbid-Jordan. e-mail: mjarrah@kfupm.edu.sa 


\section{INTRODUCTION}

Digital sinusoidal oscillators are essential elements in many applications. They are used in communications, music synthesis, control, radar, and digital signal processing. These digital oscillators exhibit the advantages of digital techniques, namely, stability, flexibility, and low cost. Moreover, the parameters of a digitally generated sinusoid are easy to control. The conventional digital sinusoidal oscillator utilizes one look-up-table in which the samples of a complete cycle of a sine wave are stored and read at appropriate time intervals $[1,2]$. The major disadvantage of a digital sinusoidal oscillator implemented using LUT method [1] is the spurious harmonic distortion due to fractional addressing of the memory $[2,3]$. The fractional addressing is necessary to increase the effective LUT length and consequently the frequency resolution of the digital sinusoidal oscillator.

The spurious harmonic distortion can be reduced by the trigonometric interpolation method [4]. Recently, two methods to reduce the spurious harmonic distortion were reported in [5]. It should be emphasized that the objective of these approaches is to reduce the complexity of the digital circuits involved measured in terms of LUT length.

In this paper, we propose three new techniques to increase the frequency resolution and at the same time reduce the spurious harmonic distortion associated with fractional addressing of the LUT. The proposed techniques have the advantage of increased frequency resolution and reduced spurious harmonic distortion over both the conventional LUT digital sinusoidal oscillator and that based on the trigonometric interpolation method. The first proposed technique, $P 1$, is based on partitioning the address register bits into three sets, namely the most significant $N_{I}$ bits, the next $N_{F 1}$ bits, and finally the least significant $N_{F 2}$ bits. The contents of the $N_{I}$ bits and $N_{F 1}$ bits are used to address LUTs as described in [4] while the content of the least significant $N_{F 2}$ bits are employed to interpolate sample values that are not stored in the LUTs. The resulting effective LUT length is $2^{N_{I}+N_{F 1}+N_{F 2}}$ while the actually needed LUT length is $2^{N_{I}}+2^{N_{F 1}+1}$. The second proposed technique, $P 2$, employs one LUT of length $2^{N_{I}}$ while the fractional bits of the address register are used to adjust the sample values in a manner which is modified version of that employed in [5]. 
The third proposed technique, $P 3$, is similar to the first technique with $N_{I}=2$ bits. The result is a reduced implementation complexity and spurious harmonic distortion.

In Section 2, we present some background and review the trigonometric and other relevant interpolation methods. In Section 3, the proposed techniques are presented and analyzed. Simulation results are presented and discussed in Section 4. Finally, the paper is concluded in Section 5.

\section{BACKGROUND AND REVIEW OF INTERPOLATION METHODS}

The conventional method for generating a digital sinusoidal signal is the well known LUT method [1]. In this method, an integer $L$ of sine wave samples are stored in a memory and then read at an appropriate time interval. The $L$ samples are taken uniformly over a full period of the sine wave. Therefore, the phase increment is $\Delta \theta=2 \pi / L$. Let $T$ be the sampling interval by which we step through the table and $i$ be the number of phase increments per step. Then, the frequency of the generated sinusoid is given by:

$$
f=\frac{i}{L T}, \quad i=1,2, \ldots, \frac{L}{2}
$$

where the maximum value of $i$ is necessary for the reconstruction of the signal and is specified by the Nyquist rate. Note that stepping through the table is simple and can be implemented by an accumulator that is incremented by $i$ every sampling interval $T$. When $i$ is restricted to integer values, the generated sinusoidal signals are of extremely low harmonic distortion. In fact, the distortion is determined by the type of number representation and number of bits, $b$, used to represent the value of a sample in the memory. For two's complement number representation with rounding, the level of harmonic distortion is approximately $2^{-2 b} / 6$ which can be brought to any desired level by increasing $b$. The frequency resolution associated with an integer increment $i$ is clearly $1 / L T$ which is fixed once $L$ and $T$ are fixed.

To increase the frequency resolution, the address register is incremented by a real number $d$ with $N_{I}$ and $N_{F}$ bits for the integer and 
fractional parts of $d$, respectively. The frequency of the generated sinusoid is, thus, given by

$$
f=\frac{d}{L T}, \quad d=2^{-N_{F}}, 2^{-N_{F}+1}, \ldots, \frac{L}{2}
$$

The frequency resolution is obviously given by

$$
\Delta f=\frac{2^{-N_{F}}}{L T}
$$

and the effective table length is $L_{e}=2^{N_{F}} L$. Because the address lines of the LUT are connected to the integer part $\left(L=2^{N_{I}}\right)$ of the address register only, the generated sinusoids have spurious harmonic distortion.

It should be noted that the spurious harmonic distortion is a consequence of truncating the content of the address register and utilizing only the integer part to address the LUT, therefore, a different sample is actually being addressed when the fractional part of the address is different from zero. Let the content of the address register at a specific instant of time be $n d=I+F$ where $I$ and $F$ are the integer and fractional parts of $n d$, respectively. The direct LUT method simply considers $I$ and neglects $F$ in addressing the LUT. The actually read sample is $\sin [\Delta \theta I]$ instead of $\sin [\Delta \theta(I+F)]$ which introduces a temporal quantization error that depends on $\Delta \theta, I$, and $F$ as given by

$$
e(\Delta \theta, I, F)=\sin [\Delta \theta I]-\sin [\Delta \theta(I+F)]
$$

The error sequence $e(\Delta \theta, I, F)$ was analyzed in [3] and shown that it reduces the signal-to-noise ratio to a value in the range $[20 \log (L)-5.17,20 \log (L)-4.92] \mathrm{dB}$. It can be shown that the error sequence $e(\Delta \theta, I, F)$ is an additive noise that causes a harmonic distortion approximately equal to $\Delta \theta^{2} / 12$ in agreement with the result in [3]. To compare between the two distortion sources let the samples in the LUT be represented by 15 bits and let $L=2048$. It follows that the word length constraint (15 bits) causes a level of harmonic distortion approximately equal to $-98 \mathrm{~dB}$ while the temporal quantization error, $e(\Delta \theta, I, F)$, results in a distortion of $-55 \mathrm{~dB}$. To avoid the distortion due to temporal quantization error, $e(\Delta \theta, I, F), L$ must be greater than $2^{17}=131072$ which is obviously a formidable 
memory size. Consequently, interpolation methods are used to reduce the amount of spurious harmonic distortion while maintaining high frequency resolution or equivalently large effective LUT length.

The trigonometric interpolation method can be summarized as follows:

$$
\sin [\Delta \theta(I+F)]=\sin [\Delta \theta I] \cos [\Delta \theta F]+\cos [\Delta \theta I] \sin [\Delta \theta F]
$$

The implementation of Eq. (5) requires two LUTs in addition to the original LUT. In particular, we must have two more LUTs for storing the sample values of $\sin [\Delta \theta F]$ and $\cos [\Delta \theta F]$. It was shown in [4] that the level of harmonic distortion associated with trigonometric interpolation method is essentially the same as that of the direct LUT method with a table length $2^{N_{F}} L$. In other words, trigonometric interpolation method increases the effective table length without practically increasing the level of harmonic distortion. The interpolation method in [5] is given by

$$
\sin [\Delta \theta(I+F)] \cong \sin [\Delta \theta I]\left\{1-\frac{(\Delta \theta F)^{2}}{2 !}\right\}+\cos [\Delta \theta I]\{\Delta \theta F\}
$$

This method has a performance comparable with that of the trigonometric interpolation method and requires less LUT length. In particular, the interpolation method in (6), denoted by $M 1$ requires only $1 / 3$ the LUT length required for trigonometric interpolation method and has essentially the same level of performance. However, the trigonometric interpolation method has low levels of harmonic distortion within an effective LUT length, $L_{e}=2^{N_{F}} L$, which is limited by the length of the used tables and can be increased only by increasing the length of these tables. On the other hand, $M 1$ exhibits low levels of harmonic distortion within the same effective LUT length and its performance is limited by the size of $L$ and $N_{F}$, therefore, $L_{e}=2^{N_{F}} L$ can be increased by increasing $N_{F}$ without the need for more memory size.

\section{THE PROPOSED TECHNIQUES}

In this section, we present three new techniques to extremely increase the frequency resolution while maintaining a reduced harmonic distortion and hardware complexity. The first proposed technique, $P 1$, 
is based on considering the fractional bits of the address register as two groups of $N_{F 1}$ and $N_{F 2}$ bits, respectively. The content of the $N_{F 2}$ bits is the least significant while the content of the integer $N_{I}$ bits is the most significant.

Let the content of the address register at a specific instant of time be $n d=I+F_{1}+F_{2}$ where $I, F_{1}$ and $F_{2}$ are the integer and fractional parts of $n d$, respectively. Hence, the value of $\sin (\Delta \theta n d)$ is given by

$$
\begin{aligned}
\sin \left[\Delta \theta\left(I+F_{1}+F_{2}\right)\right]= & \sin \left[\Delta \theta\left(I+F_{1}\right)\right] \cos \left[\Delta \theta F_{2}\right] \\
& +\cos \left[\Delta \theta\left(I+F_{1}\right)\right] \sin \left[\Delta \theta F_{2}\right]
\end{aligned}
$$

Recall that $N_{F 2}$ are the least significant bits, therefore, we can write

$$
\begin{gathered}
\sin \left[\Delta \theta F_{2}\right] \cong \Delta \theta \cdot F_{2} \\
\cos \left[\Delta \theta F_{2}\right] \cong 1
\end{gathered}
$$

The approximations in (8) and (9) are accurate enough for 15 bits word length when $N_{I}=5$ bits and $N_{F 1}=5$ bits. In this case, the maximum difference between $\sin \left[\Delta \theta F_{2}\right]$ and $\Delta \theta \cdot F_{2}$ is $3.85 \times 10^{-8}$ while the maximum difference between $\cos \left[\Delta \theta F_{2}\right]$ and 1 is $1.883 \times 10^{-5}$ which are both less than $2^{-15}$. Substituting for $\sin \left[\Delta \theta F_{2}\right]$ and $\cos \left[\Delta \theta F_{2}\right]$ from (8) and (9) in (7), we obtain

$$
\sin \left[\Delta \theta\left(I+F_{1}+F_{2}\right)\right]=\sin \left[\Delta \theta\left(I+F_{1}\right)\right]+\cos \left[\Delta \theta\left(I+F_{1}\right)\right] \cdot\left[\Delta \theta F_{2}\right]
$$

The implementation of (10) requires the generation of $\sin \left[\Delta \theta\left(I+F_{1}\right)\right]$ and $\cos \left[\Delta \theta\left(I+F_{1}\right)\right]$ as described in [4] and is illustrated by the block diagram of Figure 1. One more multiplier and an adder are needed to generate $\sin \left[\Delta \theta\left(I+F_{1}+F_{2}\right)\right]$ from $\sin \left[\Delta \theta\left(I+F_{1}\right)\right]$ and $\cos \left[\Delta \theta\left(I+F_{1}\right)\right]$. Equation (10) specifies $P 1$ which does not require any extra table length over that required by the trigonometric interpolation method. It should be noted that the implementation of Eqs. (5) and (10) require the same LUT length and that each equation require three LUTs. The first LUT is for the storage of $\sin [\Delta \theta I]$ and $\cos [\Delta \theta I]$ and is of length $2^{N_{I}}$. The second and third LUTs are of length $2^{N_{F 1}}$ each and are used for the storage of $\sin \left[\Delta \theta F_{1}\right]$ and $\cos \left[\Delta \theta F_{1}\right]$, respectively. The frequency resolution of the proposed technique is given by

$$
\Delta f=\frac{2^{-\left(N_{F 1}+N_{F 2}\right)}}{2^{N_{I}} \cdot T}
$$




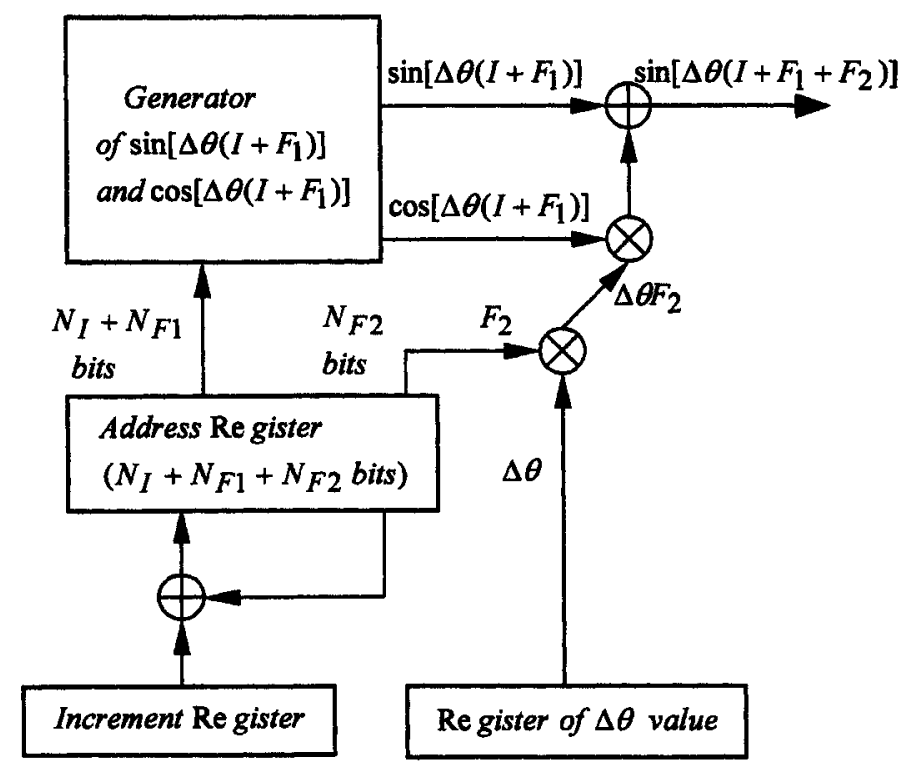

FIGURE 1 Block diagram of the first proposed technique $P 1$.

It should be noted that $P 1$ implemented with $N_{I}=N_{F 1}=5$ bits and $N_{F 2}=16$ bits has an effective LUT of $2^{26}$ with low levels of spurious harmonic distortion. The trigonometric interpolation method when implemented with $N_{I}=N_{F 1}=5$ bits has an effective LUT of $2^{10}$ with low levels of spurious harmonic distortion. When the effective LUT is increased by further increase of the fractional address length without increasing the actual LUTs lengths, the result is an increase of spurious harmonic distortion to a level comparable with that of the direct LUT method.

The second proposed technique, $P 2$, is based on modifying $M 1$ as given by

$$
\sin [\Delta \theta(I+F)] \cong \sin [\Delta \theta I]\left\{1-\frac{(\Delta \theta F)^{2}}{2 !}\right\}+\cos [\Delta \theta I]\left\{\Delta \theta F-\frac{(\Delta \theta F)^{3}}{3 !}\right\}
$$

The block diagram representation of $P 2$ is shown in Figure 2 . For $N_{I}=6$ bits, the maximum difference between $\sin [\Delta \theta F]$ and $\left\{\Delta \theta F-\left((\Delta \theta F)^{3} / 3 !\right)\right\}$ is $7.598 \times 10^{-8}$ while the maximum difference between $\cos [\Delta \theta F]$ and $\left\{1-\left((\Delta \theta F)^{2} / 2 !\right)\right\}$ is $3.869 \times 10^{-6}$ which are both less than $2^{-15}$. Consequently, the approximations in Eq. (12) are exact 


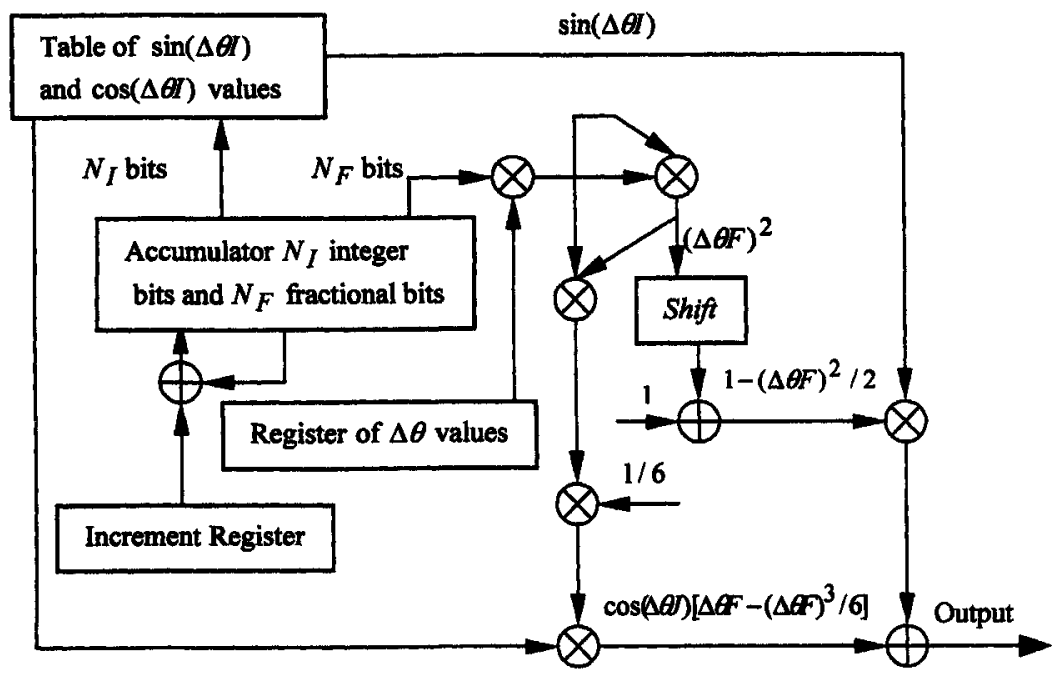

FIGURE 2 Block diagram of the second proposed method $P 2$.

for 15 bit arithmetic operations. It is evident from Eq. (12) that the implementation of $P 2$ requires only one LUT of length $L$. The associated effective LUT length and frequency resolution are $L_{e}=2^{N_{F}} L$, and $1 / L_{e} T$.

The third proposed technique, $P 3$, is based on modifying the method $P 1$ to simplify its hardware implementation. To this end, let $N_{I}=2$ bits to obtain $\Delta \theta=2 \pi / 4$, hence, $\sin [\Delta \theta I]=0, \pm 1$ and $\cos [\Delta \theta I]=0$, \pm 1 for all values of $I$. Consequently, the LUT containing the values of $\sin [\Delta \theta I]$ and $\cos [\Delta \theta I]$ can be eliminated. Furthermore, multiplication by $\sin [\Delta \theta I]$ and $\cos [\Delta \theta I]$ is a simple switching operation which can be implemented by simple logic circuit. Let the content of the address register at a specific instant of time be $n d=I+F_{1}+F_{2}$ where $I, F_{1}$ and $F_{2}$ are as defined earlier. It follows that $\sin (\Delta \theta n d)$ is given by

$$
\begin{aligned}
\sin \left[\frac{\pi}{2}\left(I+F_{1}+F_{2}\right)\right]= & \sin \left[\frac{\pi}{2}\left(I+F_{1}\right)\right] \cos \left[\frac{\pi}{2} F_{2}\right] \\
& +\cos \left[\frac{\pi}{2}\left(I+F_{1}\right)\right] \sin \left[\frac{\pi}{2} F_{2}\right]
\end{aligned}
$$

The values of $\sin \left((\pi / 2) F_{1}\right)$ and $\cos \left((\pi / 2) F_{1}\right)$ are stored in two LUTs of length $2^{N_{F 1}}$ each while the values of $\sin \left((\pi / 2) F_{2}\right)$ and $\cos \left((\pi / 2) F_{2}\right)$ 
corresponding to the least significant $N_{F 2}$ bits are approximated as given by

$$
\begin{gathered}
\sin \left(\frac{\pi}{2} F_{2}\right) \cong \frac{\pi}{2} \cdot F_{2} \\
\cos \left(\frac{\pi}{2} F_{2}\right) \cong 1-\frac{1}{2}\left(\frac{\pi}{2} \cdot F_{2}\right)^{2}
\end{gathered}
$$

The approximations in (14) and (15) are accurate enough for 15 bits word length when $N_{I}=2$ bits and $N_{F 1}=5$ bits. In this case, the maximum difference between $\sin \left((\pi / 2) F_{2}\right)$ and $(\pi / 2) \cdot F_{2}$ is $1.971 \times 10^{-5}$ while the maximum difference between $\cos \left((\pi / 2) F_{2}\right)$ and $1-(1 / 2)\left((\pi / 2) \cdot F_{2}\right)^{2}$ is $2.419 \times 10^{-7}$ which are both less than $2^{-15}$. Substituting for $\sin \left((\pi / 2) F_{2}\right)$ and $\cos \left((\pi / 2) F_{2}\right)$ from $(14)$ and (15) in (13), we obtain

$$
\begin{aligned}
\sin \left[\frac{\pi}{2}\left(I+F_{1}+F_{2}\right)\right]= & \sin \left[\frac{\pi}{2}\left(I+F_{1}\right)\right]\left\{1-\frac{1}{2}\left(\frac{\pi}{2} F_{2}\right)^{2}\right\} \\
& +\cos \left[\frac{\pi}{2}\left(I+F_{1}\right)\right] \cdot \frac{\pi}{2} \cdot F_{2}
\end{aligned}
$$

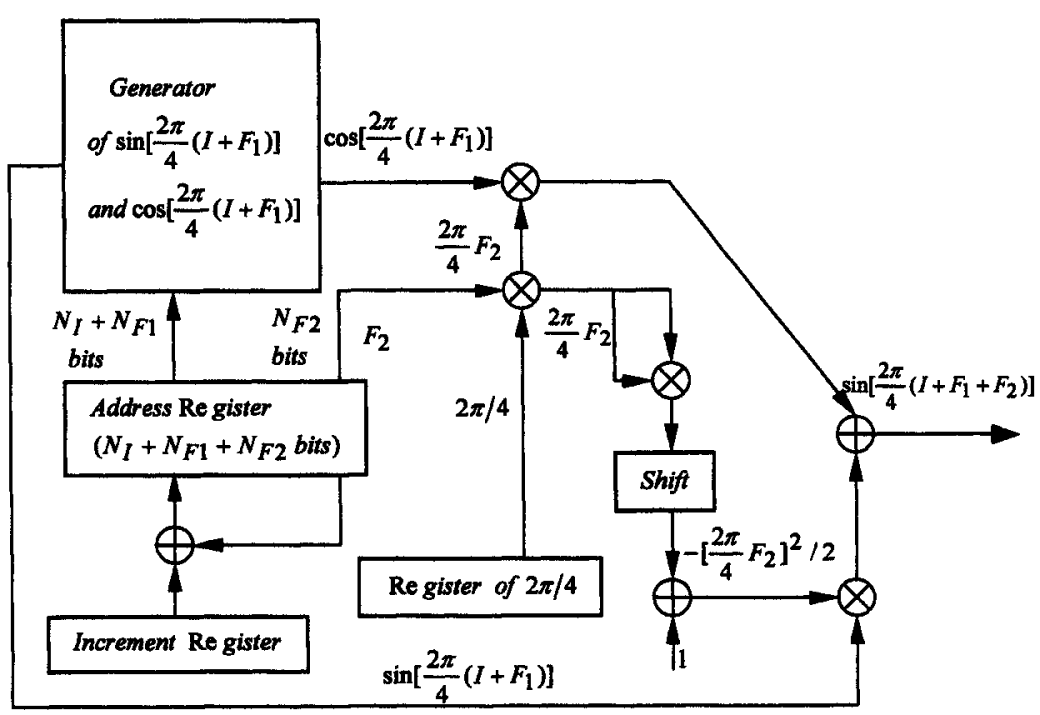

FIGURE 3 Block diagram of the third proposed technique $P 3$. 
The block diagram representation of $P 3$ is shown in Figure 3. It should be noted that $P 3$ requires less number of multipliers and LUTs than that required by $P 1$.

\section{SIMULATION RESULTS}

The direct LUT method as well as the trigonometric interpolation method and the proposed techniques are all simulated using MATLAB program. The simulation results are obtained assuming a sixteen-bit word length for all arithmetic operations. The trigonometric interpolation method and the proposed technique $P 1$ require three LUTs each containing 32 sample values while the direct LUT method is simulated using a look-up-table containing 128 sample values. The performance criterion used to compare the performance of various interpolation methods is the total harmonic distortion, $T H D$, defined by [4]

$$
\begin{aligned}
T H D & =\frac{E_{T}-E\left(f_{g}\right)}{E_{T}} \\
E_{T} & =\frac{1}{N} \sum_{k=0}^{N-1}|X(k)|^{2}
\end{aligned}
$$

where $E_{T}$ is the total energy of the sinusoidal waveform, $E\left(f_{g}\right)$ is the energy of the desired fundamental frequency $f_{g}, N$ and is the number of samples in a full period. The address increment $d$ specifies $N$ and $f_{g}=f_{c l k} / N$, where the clock frequency, $f_{c l k}$, is assumed to be $50 \mathrm{MHz}$. The address increment of the direct LUT $(L=128)$ method is $4 d$ in order to generate the same frequency of the oscillator using either the trigonometric interpolation method or the proposed technique with address increment $d$.

Table I summarizes the simulation results obtained for the level of spurious harmonic distortion associated with direct LUT method, trigonometric interpolation method, and the proposed technique $P 1$ dented by $T H D_{D}, T H D_{T}$ and $T H D_{P 1}$, respectively. The table contains the value of the address increment $d$ as varied over a wide range and the values of the generated frequency $f_{g}$. It is evident from Table I that the level of spurious harmonic distortion is the same for the three methods for integer values of address increment $d$. For fractional 
TABLE I Parameters of the oscillator based on the proposed technique $P 1$ and those using direct LUT and trigonometric interpolation methods

\begin{tabular}{lcccc}
\hline$d$ & $f g \mathrm{~Hz}$ & $T H D_{D}$ & $T H D_{T}$ & $T H D_{P 1}$ \\
\hline 1 & 1562500 & $1.770 \times 10^{-10}$ & $1.770 \times 10^{-10}$ & $1.770 \times 10^{-10}$ \\
$1 / 2$ & 781250 & $1.147 \times 10^{-10}$ & $4.322 \times 10^{-10}$ & $4.322 \times 10^{-10}$ \\
$1 / 4$ & 390625 & $1.293 \times 10^{-10}$ & $4.837 \times 10^{-10}$ & $4.837 \times 10^{-10}$ \\
$1 / 8$ & 195312.5 & $1.506 \times 10^{-4}$ & $5.302 \times 10^{-10}$ & $5.302 \times 10^{-10}$ \\
$1 / 16$ & 97656.25 & $1.882 \times 10^{-4}$ & $5.877 \times 10^{-10}$ & $5.877 \times 10^{-10}$ \\
$1 / 32$ & 48828.125 & $1.976 \times 10^{-4}$ & $6.036 \times 10^{-10}$ & $6.036 \times 10^{-10}$ \\
$1 / 64$ & 24414.06 & $2.000 \times 10^{-4}$ & $2.354 \times 10^{-6}$ & $7.329 \times 10^{-10}$ \\
$1 / 128$ & 12207.03 & $2.006 \times 10^{-4}$ & $2.942 \times 10^{-6}$ & $7.991 \times 10^{-10}$ \\
$1 / 256$ & 6103.515 & $2.007 \times 10^{-4}$ & $3.089 \times 10^{-6}$ & $8.105 \times 10^{-10}$ \\
$1 / 512$ & 3051.76 & $2.008 \times 10^{-4}$ & $3.126 \times 10^{-6}$ & $8.438 \times 10^{-10}$ \\
$1 / 1024$ & 1525.88 & $2.008 \times 10^{-4}$ & $3.135 \times 10^{-6}$ & $8.494 \times 10^{-10}$ \\
$1 / 2048$ & 762.94 & $2.008 \times 10^{-4}$ & $3.137 \times 10^{-6}$ & $8.549 \times 10^{-10}$ \\
$1 / 4096$ & 381.45 & $2.008 \times 10^{-4}$ & $3.138 \times 10^{-6}$ & $8.603 \times 10^{-10}$ \\
$1 / 8192$ & 190.73 & $2.008 \times 10^{-4}$ & $3.138 \times 10^{-6}$ & $8.601 \times 10^{-10}$ \\
\hline
\end{tabular}

values of address increment $d$ in the range $2^{-5} \leq d<1$, the performance of trigonometric interpolation method and $P 1$ is the same and is far better than that of the direct LUT method. However, for fractional values of address increment $d$ in the range $2^{-25} \leq d<2^{-5}$, the performance of trigonometric interpolation method although superior to that of the direct LUT method but is actually far less than that of $P 1$. It should be noted that the performance of trigonometric interpolation method in the range $2^{-20} \leq d<2^{-5}$ is approximately equal to that of the direct LUT method using $L=1024$. It is evident from the simulation results that for $2^{-20} \leq d<2^{-5}$, the level of $T H D_{P 1}$ associated with $P 1$ is slightly higher than its value in the range $2^{-5} \leq d<1$ and levels to a limiting value which is approximately equal to $8.6 \times 10^{-10}$.

Table II is intended to compare the performance of the interpolation method $M 1$ with that of its modified version denoted by $P 2$. It is evident from the simulation results that the performance of $P 1$ is superior to that of $M 1$ for all values of LUT length considered. The performance of $P 2$ for $L=32$ is comparable with that of $P 1$ while using only $1 / 3$ of the required memory size. When $P 2$ is simulated assuming $L=64$ and compared with $P 1$ which utilizes three tables of length 32 each (i.e., $2 / 3$ of the memory size memory size is required to implement $P 1$ ), the performance of $P 2$ is found to be superior to that of $P 1$.

Table III compares the performance of the interpolation method $P 2$ with that of $P 3$ for some typical values of $L$. It is evident from the 
TABLE II Simulation results of the oscillator based on $P 2$ and that based on the interpolation method $M 1$ for $L=32$ and $L=64$

\begin{tabular}{lllll}
\hline$d$ & $T H D_{M 1} L=32$ & $T H D_{P 2} L=32$ & $T H D_{M 1} L=64$ & $T H D_{P 2} L=64$ \\
\hline 1 & $1.770 \times 10^{-10}$ & $1.770 \times 10^{-10}$ & $1.147 \times 10^{-10}$ & $1.147 \times 10^{-10}$ \\
$1 / 2$ & $6.531 \times 10^{-9}$ & $4.322 \times 10^{-10}$ & $6.206 \times 10^{-10}$ & $2.470 \times 10^{-10}$ \\
$1 / 4$ & $4.722 \times 10^{-8}$ & $5.607 \times 10^{-10}$ & $1.253 \times 10^{-9}$ & $3.904 \times 10^{-10}$ \\
$1 / 8$ & $8.276 \times 10^{-8}$ & $5.607 \times 10^{-10}$ & $2.033 \times 10^{-9}$ & $5.260 \times 10^{-10}$ \\
$1 / 16$ & $1.049 \times 10^{-7}$ & $7.472 \times 10^{-10}$ & $2.369 \times 10^{-9}$ & $5.352 \times 10^{-10}$ \\
$1 / 32$ & $1.175 \times 10^{-7}$ & $7.542 \times 10^{-10}$ & $2.109 \times 10^{-9}$ & $5.895 \times 10^{-10}$ \\
$1 / 64$ & $1.217 \times 10^{-7}$ & $8.682 \times 10^{-10}$ & $2.383 \times 10^{-9}$ & $6.064 \times 10^{-10}$ \\
$1 / 128$ & $1.252 \times 10^{-7}$ & $8.721 \times 10^{-10}$ & $2.503 \times 10^{-9}$ & $6.099 \times 10^{-10}$ \\
$1 / 256$ & $1.269 \times 10^{-7}$ & $8.931 \times 10^{-10}$ & $2.526 \times 10^{-9}$ & $6.101 \times 10^{-10}$ \\
$1 / 512$ & $1.276 \times 10^{-7}$ & $9.303 \times 10^{-10}$ & $2.541 \times 10^{-9}$ & $6.040 \times 10^{-10}$ \\
$1 / 1024$ & $1.281 \times 10^{-7}$ & $9.124 \times 10^{-10}$ & $2.553 \times 10^{-9}$ & $6.055 \times 10^{-10}$ \\
\hline
\end{tabular}

TABLE III Simulation results of the oscillator based on $P 3$ and that based on $P 1$ for some values of $L$

\begin{tabular}{lllll}
\hline$d$ & $T H D_{P 3} L=16$ & $T H D_{P 3} L=32$ & $T H D_{P 2} L=32$ & $T H D_{P 2} L=64$ \\
\hline $1 / 8$ & $1.770 \times 10^{-10}$ & $1.770 \times 10^{-10}$ & $1.770 \times 10^{-10}$ & $1.147 \times 10^{-10}$ \\
$1 / 16$ & $1.147 \times 10^{-10}$ & $1.147 \times 10^{-10}$ & $4.322 \times 10^{-10}$ & $2.470 \times 10^{-10}$ \\
$1 / 32$ & $6.206 \times 10^{-10}$ & $1.293 \times 10^{-10}$ & $5.607 \times 10^{-10}$ & $3.904 \times 10^{-10}$ \\
$1 / 64$ & $1.253 \times 10^{-9}$ & $2.769 \times 10^{-10}$ & $5.607 \times 10^{-10}$ & $5.260 \times 10^{-10}$ \\
$1 / 128$ & $2.033 \times 10^{-9}$ & $3.883 \times 10^{-10}$ & $7.472 \times 10^{-10}$ & $5.352 \times 10^{-10}$ \\
$1 / 256$ & $2.369 \times 10^{-9}$ & $4.873 \times 10^{-10}$ & $7.542 \times 10^{-10}$ & $5.895 \times 10^{-10}$ \\
$1 / 512$ & $2.109 \times 10^{-9}$ & $5.510 \times 10^{-10}$ & $8.682 \times 10^{-10}$ & $6.064 \times 10^{-10}$ \\
$1 / 1024$ & $2.383 \times 10^{-9}$ & $5.813 \times 10^{-10}$ & $8.721 \times 10^{-10}$ & $6.099 \times 10^{-10}$ \\
$1 / 2048$ & $2.503 \times 10^{-9}$ & $5.789 \times 10^{-10}$ & $8.931 \times 10^{-10}$ & $6.101 \times 10^{-10}$ \\
$1 / 4096$ & $2.526 \times 10^{-9}$ & $5.944 \times 10^{-10}$ & $9.303 \times 10^{-10}$ & $6.040 \times 10^{-10}$ \\
$1 / 8192$ & $2.541 \times 10^{-9}$ & $5.991 \times 10^{-10}$ & $9.124 \times 10^{-10}$ & $6.055 \times 10^{-10}$ \\
\hline
\end{tabular}

simulation results that the performance of $P 3$ is superior to that of $P 2$ for all values of LUT length considered.

The advantages of the proposed techniques over the direct LUT and trigonometric interpolation methods are clear. In particular, the proposed techniques outperform all other interpolation methods with essentially the same level of hardware complexity. Moreover, their performance is essentially the same over an extremely wide frequency range with extremely high frequency resolution. In fact the frequency ranges of $P 1, P 2$ and $P 3$ are limited by the number of bits used to implement the fractional part of the address register rather than being limited by the look-up-table length. Therefore, by increasing the width of the fractional part of the address register, we can practically achieve any desired level of frequency resolution at the specified level of spurious harmonic distortion. 


\section{CONCLUSION}

Three new techniques are proposed to significantly increase the frequency resolution and reduce the amount of spurious harmonic distortion in digital sinusoidal oscillators using Look-upTable method. The proposed methods are simulated by a MATLAB program on a general purpose computer. The simulation results show that the harmonic distortion of the proposed methods is significantly less than that of both direct LUT and trigonometric interpolation methods for all cases in which the content of the least significant $N_{F 2}$ bits is different from zero. Moreover, the performance of the first proposed method, $P 1$, is identical to that of the trigonometric interpolation method for all cases in which the content of the least significant $N_{F 2}$ bits is zero. The performance of the third proposed method, $P 3$, is superior to that of $P 1, P 2$, and the trigonometric interpolation method. The proposed techniques are suitable for implementation on already available digital signal processors like TMS32010 DSP. In addition, digital sinusoidal oscillators based on the proposed methods can be fabricated on a single chip using monolithic large-scale integration.

\section{Acknowledgment}

The author would like to acknowledge the support provided by King Fahd University of Petroleum and Minerals.

\section{References}

[1] Garcia, D. (1986). "Precision digital sine wave generation with the TMS32010", In: Digital Signal Processing Applications with the TMS320 Family. Dallas, TX: Texas Instruments, pp. 269-289.

[2] Jeng, Y. C., "Digital spectra of nonuniformly sampled signals: Fundamentals and high-speed waveform digitizers", IEEE Transactions on Instrumentation and Measurement, IM-37, 245-251, June, 1988.

[3] Jeng, Y. C., "Digital spectra of nonuniformly sampled signals: Digital look-up tunable sinusoidal oscillator", IEEE Transactions on Instrumentation and Measurement, IM-37, 358-362, Sept., 1988.

[4] Schanerberger, M. and Awad, S. S., "The implementation of digital sine wave oscillator using the TMS320C25: Distortion reduction and applications", IEEE Transactions on Instrumentation and Measurement, IM-39, 870-873, Dec., 1990.

[5] Al-Ibrahim, M. M. and Bataineh, S., "High resolution and low distortion digital look-up-table sinusoidal oscillators", International Journal of Electronics, 87(1), January, 2000. 

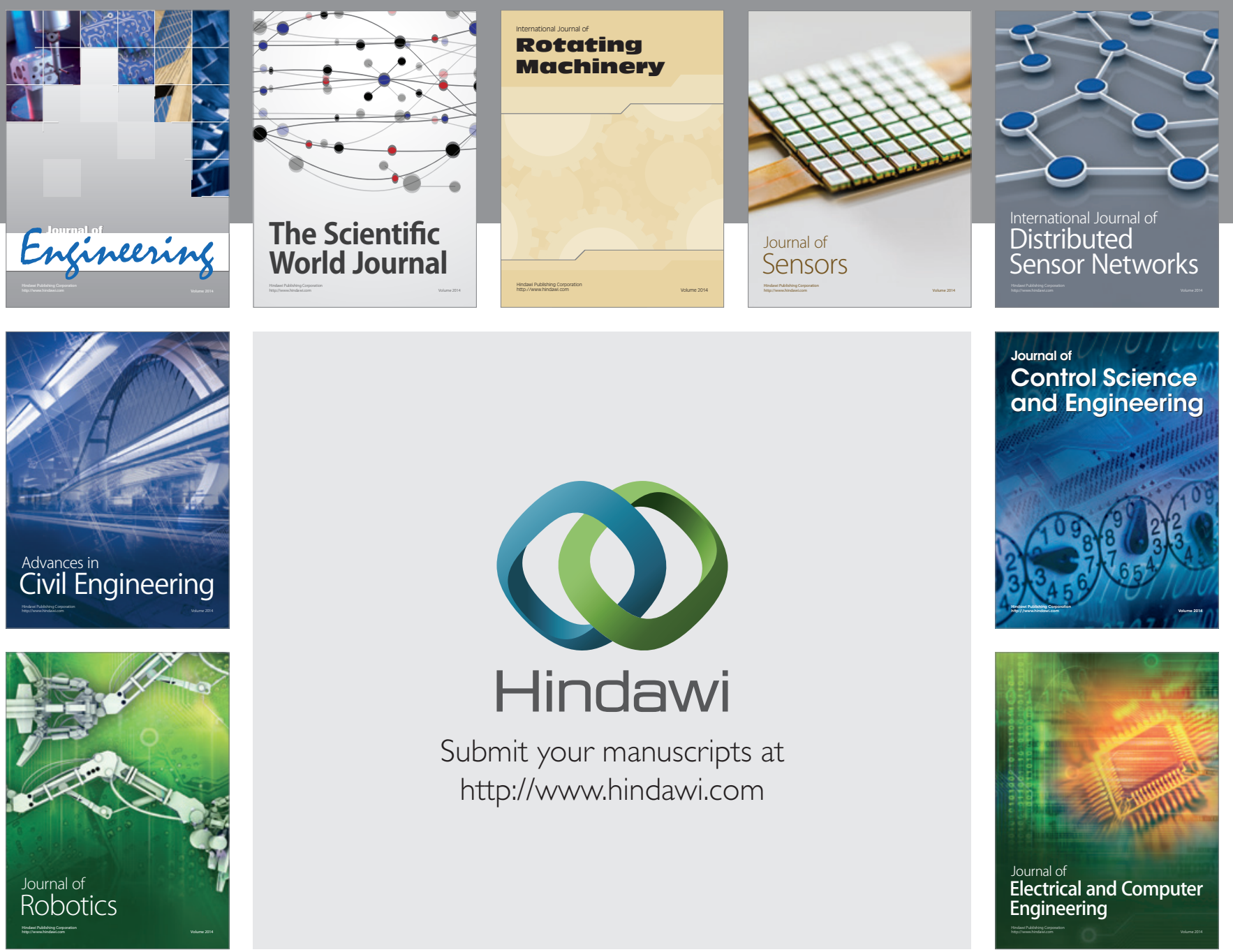

Submit your manuscripts at

http://www.hindawi.com
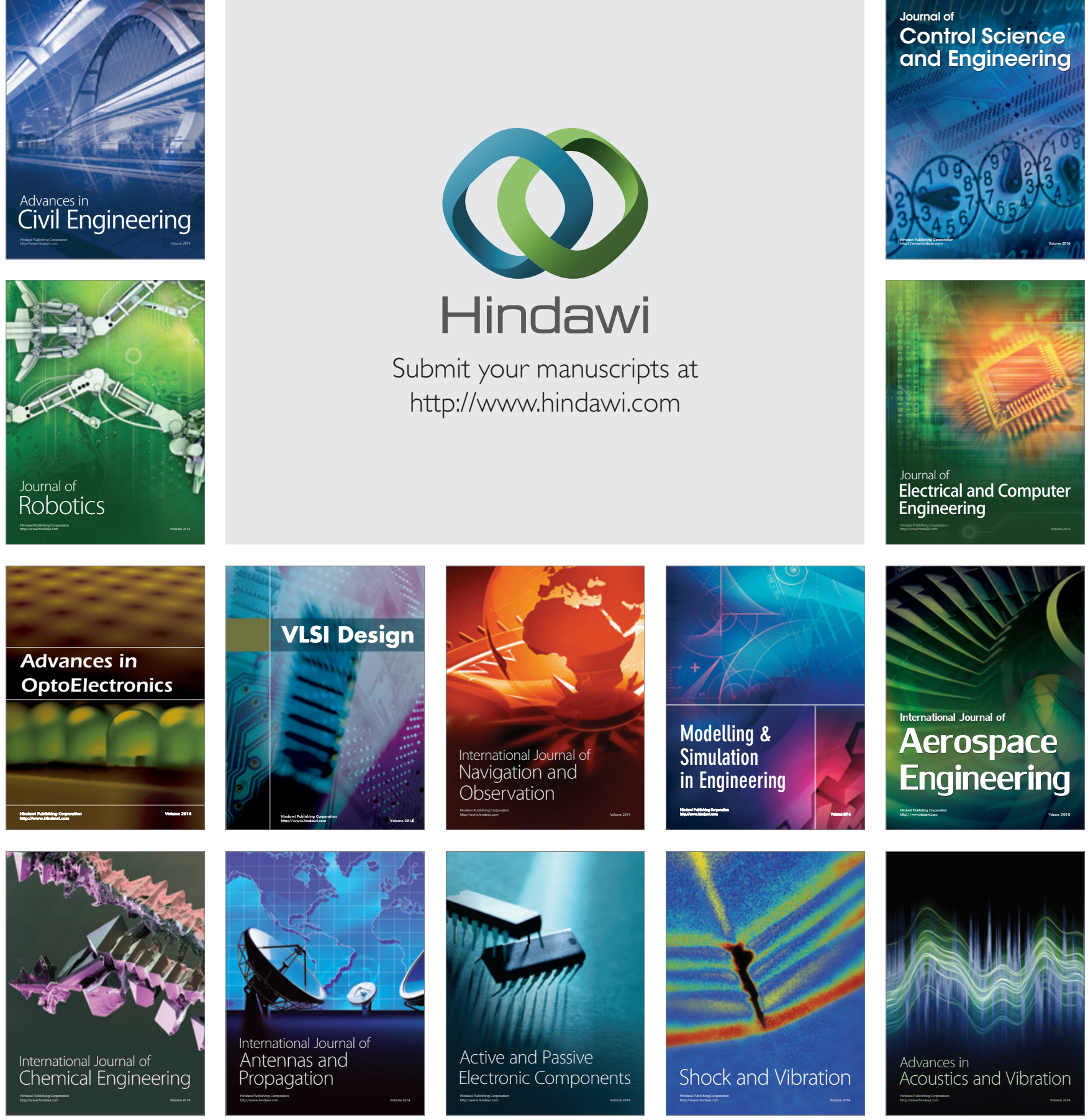